\title{
Higher-order Aspects of Logic Programming
}

\author{
Uday S. Reddy \\ Department of Computer Science \\ University of Mlinois at Urbana-Champaign \\ Urbana, IL 61801 \\ Net: reddy@cs.uiuc.edu
}

\begin{abstract}
Are higher-order extensions to logic programming needed? We suggest a negative answer by showing that higher-order features are already available in pure logic programming. It is demonstrated that higher-order lambda calculus-based languages can be compositionally embedded in logic programming languages preserving their semantics and abstraction facilities. Further, we show that such higher-order techniques correspond to programming techniques often practiced in logic programming.

Keywords: Higher-order features, functional programming, lambda calculus, logic variables, concurrent logic programming, types, semantics.
\end{abstract}

\section{Introduction}

In an early paper [War82], Warren raised the question whether higher-order extensions to Prolog were necessary. He suggested that they were not necessary by modelling higher-order concepts in logic programs. His proposal modelled functions and relations intensionally by their names. As is well-known in Lisp community, such an encoding does not obtain the full power of higher-order programming. In particular, lambda abstraction and "upward" function values are absent.

An entirely different approach to the issue was pursued by Miller et. al. in Lambda Prolog [MN86]. The objective here seems to be not higher-order logic programming, but logic programming over higher-order terms. While this approach has many interesting applications, particularly in meta-logical frameworks, it still leaves open the question of whether higher-order programming is possible in a standard logic programming setting. A similar comment applies to other higher-order languages like HiLog [CKW89].

In this paper, we return to Warren's question and suggest another negative answer. Using new insights obtained from the connection between linear logic and logic programming [Laf87, Abr91, Abr93, Red93b], we contend that idealized logic programming is "already higher-order" in its concept, even though one might wish to add some syntactic sugar to obtain convenience. A similar answer is also suggested by Saraswat's work [Sar92] showing that concurrent logic programming is higher-order in a categorical sense.

We argue that idealized logic programming is higher-order by presenting two forms of evidence: 
- We exhibit a compositional, semantics-preserving translation from higherorder lambda calculus-based languages to logic programs.

- We show that higher-order programming techniques are already in use in logic programming (albeit in a limited form) and that these can be generalized.

Two features of logic programming play important role in this modelling: logic variables and concurrency. Using logic variables, one often constructs data structures with multiple occurrences of variables, e.g., difference lists [CT77]. Typically, one of the occurrences of the variable is designated for input, i.e., a binding is given to the variable via this occurrence, and other occurrences are used as output. If we form a pair $\left(x, S_{x}\right)$ consisting of a variable $x$ and a structure $S_{x}$ with embedded occurrences of $x$ (see Fig. 1), we have essentially formed a "function" value. The function is "applied" to an argument by binding the variable $x$ and the result of application is obtained via $S_{x}$. Whereas this scheme allows $S_{x}$ to be a data structure, using concurrency, one can similarly build computational structures with shared variables. $S_{x}$ is then thought of as a "process" and a pair of variables involved in the process is viewed as a function value. All this provides "functions" that are good for only one function application. General functions that can be used multiple times are modelled by generating entire streams of such pairs, again using a process-oriented view. The streams are generated in a demand-driven fashion so that the user of the stream can terminate the stream at will. Mathematically, such streams are best viewed as finite sets.

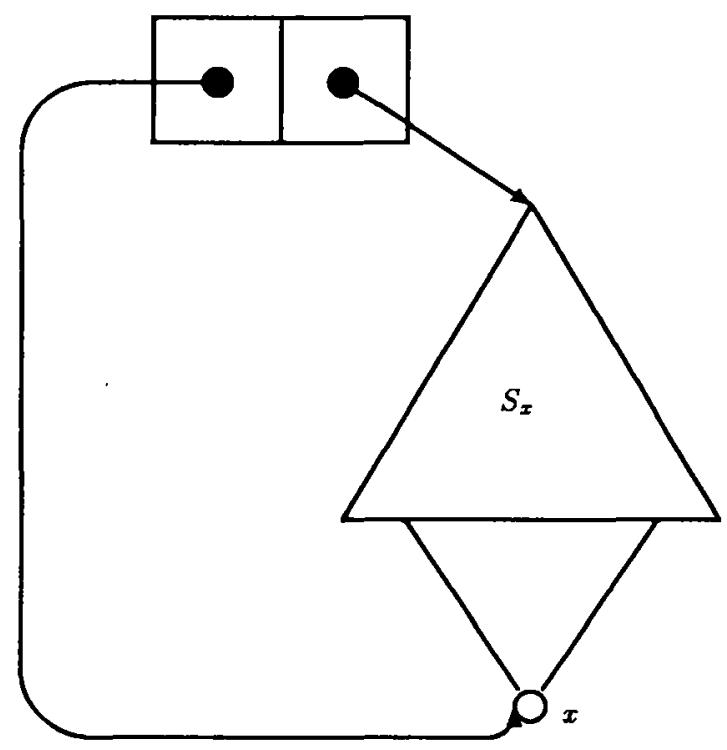

Fig. 1. Difference structures 
Concurrency is often thought of as an extraneous feature of logic programming. However, idealized logic programs executed with fair SLD-resolution [LM84] are necessarily concurrent. Under a fair resolution scheme, no goal can be delayed forever. So, each goal of a query must be thought of as a separate process which cooperates with other processes to solve the problem. Our modelling of higher-order concepts is in this framework. This cannot be duplicated in sequential logic programming languages like Prolog due to the crucial role of concurrency already mentioned. However, our scheme is applicable to almost all concurrent logic programming languages such as Concurrent Prolog [Sha83], Parlog [Gre87] and Guarded Horn clauses [Ued86] as well as languages with coroutine facilities like Mu-Prolog [Nai86].

\subsection{Related work}

From a theoretical point of view, the fact that higher-order functions can be simulated by relations seems to have been folklore in category theory for some time. See [Bar91] for an explicit presentation. Within the programming language theory, it is implicit in the work of [Laf87, Abr91, Red93b] which shows that linear logic proofs can be modelled as logic programs. Another piece of related work is Milner's embedding of lambda calculus in pi-calculus [Mil90]. His embedding only uses a subset that has a direct correspondence to concurrent logic programming languages. Thus, our presentation documents what has been implicitly known in various programming language circles.

Many of our ideas are also implicit in Shapiro's work on modelling objects in concurrent logic programming [Sha83, ST86]. Since objects provide collections of operations to their clients, modelling objects involves modelling function values as well. In fact, as shown in [Red93a], our translations easily extend to higherorder imperative programs (object-based programs) using the same ideas as Shapiro's. Somewhat more explicit is the work of Saraswat [Sar92] where it is shown in that the semantic model of concurrent constraint programs forms a cartesian closed category. In contrast to this work, we show here that standard logic programming has the same kind of properties.

\section{The framework}

We find it convenient to treat logic programs as defining binary relations. So, a predicate definition looks like:

$$
\begin{aligned}
& R: A \leftrightarrow B \\
& R(x, y) \Leftrightarrow \psi
\end{aligned}
$$

where $A$ and $B$ are types, and $\psi$ is a formula built from predicate applications, equality, conjunction, disjunction and existential quantification. (We often suppress the existential quantifiers and disjunctions by using Horn clause notation 
$R(x, y) \leftarrow \psi)$. Typically, the type $A$ is a finite product type, in which case we write:

$$
\begin{aligned}
& R: A_{1} \times \ldots \times A_{k} \leftrightarrow B \\
& R\left(x_{1}, \ldots, x_{k}, y\right) \Leftrightarrow \psi
\end{aligned}
$$

Note that we suppress the parentheses around the tuple $\left(x_{1}, \ldots, x_{k}\right)$, to avoid clutter. The special case of $k=0$ appears as:

$$
\begin{aligned}
& R: \text { unit } \leftrightarrow B \\
& R(y) \Leftrightarrow \psi
\end{aligned}
$$

Types include

- primitive types such as int and bool,

- finite products, including the nullary product called unit, and

- finite disjoint unions, including the nullary version $\emptyset$.

We often write disjoint unions with constructor symbols for injections, e.g.,

$$
\text { type result }=\text { fail }(\text { unit })+\operatorname{succ}(i n t)
$$

which is syntactic sugar for unit + int. All symbols other than constructors of this kind are variables (usually $x, y, z$ and $X, Y, Z$ ). We do not use a case convention for variables. We also allow polymorphic type constructions with recursive definitions, e.g.,

$$
\text { type } \operatorname{list}(\alpha)=\operatorname{nil}(\text { unit })+\alpha \cdot \operatorname{list}(\alpha)
$$

which denotes the least set $L$ such that $L=u n i t+\alpha \times L$. All these constructions have well-defined semantics. See, for example, [LR91].

\section{Embedding PCF}

PCF (Programming Language for Computable Functions), due to Scott [Sco69], is a higher-order functional programming language. (See [Plo77] and [Gun92, 4.1] for published descriptions.) PCF is a typed lambda calculus with a single primitive type for integers. (The boolean type can be omitted without loss of expressiveness.) In this section, we show how PCF can be embedded in a logic programming language. The modelling of functions being the crux of the problem, extension to other kinds of data structures is more or less immediate.

Examples We start by showing some examples. First, consider "linear" functions, i.e., functions that use their arguments exactly once. This restriction allows us to introduce a simpler model first. Here is an example:

$$
\begin{array}{ll}
\text { inc : int } \triangleright \text { int } & \text { inc : int } \leftrightarrow \text { int } \\
\operatorname{inc}(x)=x+1 & \text { inc }(x, y) \Leftrightarrow \operatorname{add}(x, 1, y)
\end{array}
$$


On the left, we have a term $x+1$ treated as a function of $x$. We always use the symbol $\triangleright$ to denote this kind of implicit function that occurs at the top level. This function is modelled as a relation between integers. Such modelling of functions by relations is elementary for any one with some knowledge of set theory.

In lambda calculus, one can abstract over $x$ to form a function term:

$$
\begin{array}{ll}
\text { succ }: \triangleright \text { int } \rightarrow \text { int } & \text { succ }: \text { unit } \leftrightarrow(\text { int } \times \text { int }) \\
\text { succ }=\lambda \mathbf{x} \cdot \mathrm{x}+1 & \operatorname{succ}((\mathrm{x}, \mathrm{y})) \Leftrightarrow \operatorname{add}(\mathrm{x}, 1, \mathrm{y})
\end{array}
$$

The function space int $\rightarrow$ int is modelled by the product int $\times$ int. If this seems surprising, consider the fact that a relation $A \leftrightarrow B$ is really like a function $A \rightarrow \mathbb{P} B$. Due to the implicit power set construction, smaller cardinalities suffice in the second argument position of the relation. The point of this example is to illustrate that lambda-abstraction is modelled by pair formation in a logic program. This is the same kind of pair formation as that shown in Fig. 1 except that the "difference" between the input and output is a computation rather than a data structure.

A generic function composition term is modelled as follows:

$$
\begin{aligned}
\text { compose }:(A \rightarrow & B) \times(B \rightarrow C) \triangleright(A \rightarrow C) \\
\text { compose }(\mathrm{f}, \mathrm{g})= & \lambda \mathrm{x} . \mathrm{g}(\mathrm{f} \mathrm{x}) \\
& \text { compose }:(A \times B) \times(B \times C) \leftrightarrow(A \times C) \\
& \text { compose }(\mathrm{f}, \mathrm{g},(\mathrm{x}, \mathrm{z})) \leftarrow \mathrm{f}=(\mathrm{x}, \mathrm{y}), \mathrm{g}=(\mathrm{y}, \mathrm{z})
\end{aligned}
$$

Notice that a function application, such as $f x$, is modelled by pair decomposition: $f=(x, y)$. We can use compose to make a twice composition of the succ function as follows:

$$
\operatorname{succ}(f), \operatorname{succ}(g), \operatorname{compose}(f, g, h)
$$

Note that $h$ denotes the required composition: $h=(x, z)$ if and only if $z=x+2$. This would correspond to the PCF term compose (succ, succ) $=\lambda x \cdot\left(\lambda x^{\prime} \cdot x^{\prime}+1\right)$ $\left(\left(\lambda x^{\prime} \cdot x^{\prime}+1\right) x\right)$. Abstraction over $f$ and $g$ yields a composition "function" as a term:

$$
\begin{array}{r}
\text { comp : } \begin{array}{r}
\text { comp }=\lambda \mathrm{f} . \lambda \mathrm{g} . \lambda \mathrm{x} \cdot \mathrm{g}(\mathrm{f} \mathrm{x}) \\
\operatorname{com}((B \rightarrow C) \rightarrow(A \rightarrow C)) \\
\\
\quad \operatorname{comp}: \text { unit } \leftrightarrow(A \times B) \times((B \times C) \times(A \times C)) \\
\operatorname{comp}((\mathrm{f},(\mathrm{g},(\mathrm{x}, \mathrm{z})))) \leftarrow \mathrm{f}=(\mathrm{x}, \mathrm{y}), \mathrm{g}=(\mathrm{y}, \mathrm{z})
\end{array}
\end{array}
$$

Consider the PCF term comp succ. This denotes a higher-order function which, when applied to any function $g:$ int $\rightarrow$ int, yields the function $\lambda x \cdot g(x+1)$. Its effect is obtained by the logic programming query:

$$
\operatorname{succ}(\mathbf{f}), \operatorname{comp}(\mathbf{f}, \mathbf{h})
$$

Note that $h=(g,(x, z))$ if and only if $g=(x+1, z)$. Thus, the above scheme of translation works for functions of any order as long as the arguments of functions are used linearly. 
Control Let us pause to examine the control issues. A query involving higherorder values, e.g., $\operatorname{succ}(f), \operatorname{succ}(g)$, compose $(f, g, h), h=(1, z)$, must not be evaluated sequentially. Notice that $\operatorname{succ}(f)$ reduces to $\operatorname{add}(x, 1, y)$ which has an infinite number of solutions. The best order of evaluation would be the following:

$$
\begin{aligned}
& \leftarrow \operatorname{succ}(\mathbf{f}), \operatorname{succ}(\mathrm{g}), \operatorname{compose}(\mathrm{f}, \mathrm{g}, \mathrm{h}), \mathrm{h}=(1, \mathrm{z}) \\
& \leftarrow \operatorname{succ}(\mathbf{f}), \operatorname{succ}(\mathrm{g}), \operatorname{compose}(\mathrm{f}, \mathrm{g},(1, \mathrm{z})) \\
& \leftarrow \operatorname{succ}(\mathrm{f}), \operatorname{succ}(\mathrm{g}), \mathrm{f}=(1, \mathrm{y}), \mathrm{g}=(\mathrm{y}, \mathrm{z}) \\
& \leftarrow \operatorname{succ}((1, \mathrm{y})), \operatorname{succ}((\mathrm{y}, \mathrm{z})) \\
& \leftarrow \operatorname{add}(1,1, \mathrm{y}), \operatorname{add}(\mathrm{y}, 1, \mathrm{z}) \\
& \leftarrow \operatorname{add}(2,1, \mathrm{z})
\end{aligned}
$$

This order of evaluation is achieved if

- every goal literal is suspended until it matches (becomes an instance of) the head of a clause, and

- literals of primitive predicates, like add, are suspended until their input arguments are instantiated.

In essence, we wish to treat our clauses as Guarded Horn clauses [Ued86]. While most of our clauses have empty guards, the translation of conditional expressions will involve guards as well. It is interesting that the translation of functional programs should automatically give rise to GHC programs.

General functions To model general functions, we must handle multiple uses of arguments. Keeping in mind that arguments are often themselves functions, we model such multiple-use arguments as finite sets of values. For example, here is the twice function that composes its argument with itself:

$$
\begin{aligned}
\text { twice }:(A \rightarrow A) & \triangleright(A \rightarrow A) \\
\text { twice }(\mathrm{f})=\lambda \mathbf{x} . \mathrm{f}(\mathrm{f} \mathbf{x}) & \\
& \text { twice }: \mathbb{F}(A \times A) \leftrightarrow(A \times A) \\
& \text { twice }(\mathbf{F},(\mathbf{x}, \mathrm{z})) \leftarrow \mathbf{F}=\{\mathrm{f} 1, \mathrm{f} 2\}, \mathrm{f} 1=(\mathrm{x}, \mathrm{y}), \mathrm{f} 2=(\mathrm{y}, \mathrm{z})
\end{aligned}
$$

Here, the input to the twice relation is a finite set of pairs, i.e., a finite piece of a function graph. The twice function extracts two (not necessarily distinct) pairs from such a piece.

How do we make such pieces of function graphs? We first illustrate it for the succ function:

$$
\begin{aligned}
& m k s u c c: \text { unit } \leftrightarrow \mathbb{F}(\text { int } \times \text { int }) \\
& \operatorname{mksucc}(\{\}) \leftarrow \\
& \operatorname{mksucc}(\{(x, y)\}) \leftarrow \operatorname{add}(x, 1, y) \\
& \operatorname{mksucc}(\mathrm{F} 1 \cup \mathrm{F} 2) \leftarrow \operatorname{mksucc}(\mathrm{F} 1), \operatorname{mksucc}(\mathrm{F} 2)
\end{aligned}
$$

Note that mksucc makes as many pairs of the succ function as needed. In particular, the query $m k s u c c(F), F=\{f 1\} \cup\{f 2\}$ is equivalent to: 


$$
\mathrm{f} 1=(\mathrm{x} 1, \mathrm{y} 1), \operatorname{add}(\mathrm{x} 1,1, \mathrm{y} 1), \mathrm{f} 2=(\mathrm{x} 2, \mathrm{y} 2), \operatorname{add}(\mathrm{x} 2,1, \mathrm{y} 2)
$$

Now, we can create the twice composition of the succ function by the following simpler goal:

$$
\operatorname{mksucc}(F), \text { twice(F, h) }
$$

which corresponds to the PCF term twice(succ). If we would like to use one portion of the succ function graph for use with twice and keep the rest for other purposes, that is easy enough to do too:

$$
\left.\operatorname{mksucc}\left(F \cup F^{\prime}\right) \text {, twice( } F, h\right), \ldots F^{\prime} \ldots
$$

We now generalize the idea involved in $m k s u c c$ to make finite portions of any graph. Notice that the relations of interest to us are, in general, of the form:

$$
\begin{aligned}
& R: A_{1} \times \ldots \times A_{n} \leftrightarrow C \\
& R(\bar{x}, u) \leftarrow \bar{x}=\bar{t}, \psi
\end{aligned}
$$

The terms $\bar{t}$ decompose the inputs $\bar{x}$, the goal $\psi$ performs some computation using the inputs and $u$ is the output term. We assume that existential quantification is used in $\psi$ such that $F V(\psi) \subseteq F V(\bar{t}) \cup F V(u)$. We can denote such relations compactly as input-computation-output triples written as $\bar{t}|\psi| u$.

Suppose $R=(\bar{t}|\psi| u)$ is a relation of type $\mathbb{F} A_{1} \times \ldots \times \mathbb{F} A_{n} \leftrightarrow C$. Then, define a generic definition scheme (or "macro") for making graphs of the outputs as follows:

$$
\begin{aligned}
& \operatorname{mkgraph}[\bar{t}|\psi| u]: \mathbb{F} A_{1} \times \ldots \times \mathbb{F} A_{n} \leftrightarrow \mathbb{F} C \\
& \text { mkgraph }[\bar{t}|\psi| u]=P \text { where } \\
& \quad P(\bar{x},\{\}) \leftarrow \bar{x}=\{\} \\
& \quad P(\bar{x},\{u\}) \leftarrow \bar{x}=\bar{t}, \psi \\
& \quad P\left(\bar{x}, y_{1} \cup y_{2}\right) \leftarrow \bar{x}=\bar{x}_{1} \cup \bar{x}_{2}, P\left(\bar{x}_{1}, y_{1}\right), P\left(\bar{x}_{2}, y_{2}\right)
\end{aligned}
$$

Here, $\vec{x}=\{\}$ as well as $\bar{x}=\bar{x}_{1} \cup \bar{x}_{2}$ mean their component-wise expansions.

Using the mkgraph scheme, we can represent the translations of PCF terms compactly. For example:

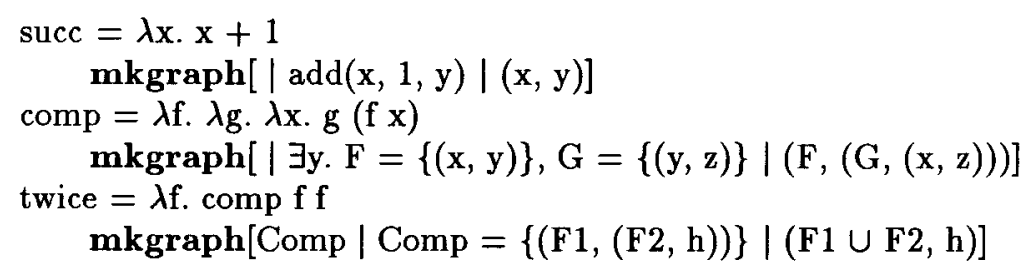

One is often tempted to ask the somewhat meaningless question, "how many elements are there in a finite set produced by an instance of mkgraph"? Well, the answer is "as many as needed". A mkgraph predicate is able to produce all possible subsets of a graph. Treating its clauses as guarded Horn clauses allows the predicate to be suspended until a client process determines the surface structure of the finite set. The mkgraph predicate then fills in the elements of the finite set. 
Finite sets Our translation makes use of a finite set type which is not usually in the standard repertoire of logic programming. However, the unification problem for finite set-terms is decidable and it is certainly possible to add finite sets to a logic programming language. (See, for example, [DOPR91].)

On the other hand, a more modest method suffices for the purposes of this paper. Our use of finite sets is only via the mkgraph scheme and, within the scheme, a set constructor always appears with variable arguments. Therefore, the general set-unification algorithm is not needed. We can treat finite sets as a constructor data type of the following form:

$$
\text { type } \mathbb{F}(\alpha)=\text { null }+\operatorname{single}(\alpha)+\operatorname{union}(\mathbb{F}(\alpha), \mathbb{F}(\alpha))
$$

The symbols null, single and union are constructors for finite sets. For readability, we continue to write $n u l l$ as \{\} , single $(t)$ as $\{t\}$, and $u n i o n(X, Y)$ as $X \cup Y$. We also use the short hand $\left\{t_{1}, \ldots, t_{n}\right\}$ for $\left\{t_{1}\right\} \cup \ldots \cup\left\{t_{n}\right\}$.

This simplification would be valid only if our use of the above constructor terms is consistent with the properties of finite sets. We verify such consistency as follows. Let $\equiv$ be the least congruence relation generated by the following theory of finite sets:

$$
\begin{aligned}
\operatorname{union}(X, \operatorname{null}) & \equiv X \\
\text { union }(\text { null, }, X) & \equiv X \\
\operatorname{union}\left(X_{1}, \operatorname{union}\left(X_{2}, X_{3}\right)\right) & \equiv \operatorname{union}\left(\operatorname{union}\left(X_{1}, X_{2}\right), X_{3}\right) \\
\operatorname{union}\left(X_{1}, X_{2}\right) & \equiv \operatorname{union}\left(X_{2}, X_{1}\right) \\
\operatorname{union}(X, X) & \equiv X
\end{aligned}
$$

Then:

Proposition 1. Let $P: \mathbb{F} A_{1} \times \ldots \times \mathbb{F} A_{k} \leftrightarrow \mathbb{F} C$ be an instance of the mkgraph scheme, and $y_{1}, y_{2} \in \mathbb{F} C$ such that $y_{1} \equiv y_{2}$. Then, for all $\bar{x}_{1} \in \mathbb{F} A_{1} \times \ldots \times \mathbb{F} A_{k}$ such that $P\left(\bar{x}_{1}, y_{1}\right)$, there exists $\bar{x}_{2} \in \mathbb{F} A_{1} \times \ldots \times \mathbb{F} A_{k}$ such that $\bar{x}_{1} \equiv \bar{x}_{2}$ and $P\left(\bar{x}_{2}, y_{2}\right)$.

Proof. By induction on the derivation of $y_{1} \equiv y_{2}$. We show two of the base cases. The remaining cases are similar.

- If $y_{1}=Y \cup\{\}$ and $y_{2}=Y$ then $\bar{x}_{1}=\bar{X} \cup\{\}$, for some $\bar{X}$, and $P(\bar{X}, Y)$. Let $\bar{x}_{2}=\bar{X}$.

- If $y_{1}=Y \cup Y^{\prime}$ and $y_{2}=Y^{\prime} \cup Y$ then $\bar{x}_{1}=\bar{X} \cup \bar{X}^{\prime}$, for some $\bar{X}, \bar{X}^{\prime}$, and $P(\bar{X}, Y)$ and $P\left(\bar{X}^{\prime}, Y^{\prime}\right)$. Let $\bar{x}_{2}=\bar{X}^{\prime} \cup \bar{X}$.

For the induction step of monotonic extension, let $y_{1}^{\prime} \equiv y_{2}^{\prime}$ and $y_{1}^{\prime \prime} \equiv y_{2}^{\prime \prime}$. If $\bar{x}_{1} \in \mathbb{F} A_{1} \times \ldots \times \mathbb{F} A_{k}$ such that $P\left(\bar{x}_{1}, y_{1}^{\prime} \cup y_{1}^{\prime \prime}\right)$ then $\bar{x}_{1}=\bar{x}_{1}^{\prime} \cup \bar{x}_{1}^{\prime \prime}, P\left(\bar{x}_{1}^{\prime}, y_{1}^{\prime}\right)$ and $P\left(\bar{x}_{1}^{\prime \prime}, y_{1}^{\prime \prime}\right)$ for some $\bar{x}_{1}^{\prime}$ and $\bar{x}_{1}^{\prime \prime}$. Then, by induction hypothesis, there exist $\bar{x}_{2}^{\prime}$ and $\bar{x}_{2}^{\prime \prime}$ such that $\bar{x}_{1}^{\prime} \equiv \bar{x}_{2}^{\prime}, \bar{x}_{1}^{\prime \prime} \equiv \bar{x}_{2}^{\prime \prime}, P\left(\bar{x}_{2}^{\prime}, y_{2}^{\prime}\right)$ and $P\left(\bar{x}_{2}^{\prime \prime}, y_{2}^{\prime \prime}\right)$. Let $\bar{x}_{2}=\bar{x}_{2}^{\prime} \cup \bar{x}_{2}^{\prime \prime}$. 
Another method, available in a committed-choice language, is to represent finite sets by streams. Union is then obtained by merge. Here is the definition of the mkgraph scheme using this representation:

$$
\begin{aligned}
& \operatorname{mkgraph}[\bar{t}|\psi| u]: \operatorname{list}\left(A_{1}\right) \times \ldots \operatorname{list}\left(A_{n}\right) \leftrightarrow \operatorname{list}(C) \\
& \operatorname{mkgraph}[\bar{t}|\psi| u]=P \text { where } \\
& \quad P(\bar{x},[]) \leftarrow \bar{x}=[] \\
& \quad P(\bar{x}, u . y s) \leftarrow m e r g e\left(\bar{x}_{1}, \bar{x}_{2}, \bar{x}\right), \bar{x}_{1}=\bar{t}, \psi, P\left(\bar{x}_{2}, y s\right)
\end{aligned}
$$

This makes no essential change to the mkgraph scheme except for linearizing the recursion. We can profitably use Shapiro's metaphor [Sha83] and think of a mkgraph predicate as an "object" and the finite set argument as a stream of "messages" sent to the object.

\subsection{Translation}

Table 1 gives a description of PCF types, terms and the type rules for terms. The type rules are expressed in terms of judgements of the form

$$
x_{1}: A_{1}, \ldots, x_{n}: A_{n} \triangleright M: C
$$

where $x_{1}, \ldots, x_{n}$ are the free variables of $M$. We use $\Gamma, \Delta, \ldots$ to range over sequences of variable typings $x_{1}: A_{1}, \ldots, x_{n}: A_{n}$ (called "type contexts"). All the variables in a type context must be distinct. The first type rule (called Exchange rule) allows the typings in a type context to be freely rearranged. So, the order of typings is essentially insignificant. The type rules of Table 1 look different from the usual formulations, but they are equivalent to them. They are designed to give the cleanest possible translation.

The translation of PCF programs to logic programs is as follows. The types are translated by the mapping ()$^{\circ}$ :

$$
\begin{aligned}
\text { int }^{\circ} & =\text { int } \\
(A \rightarrow B)^{\circ} & =\mathbb{F} A^{\circ} \times B^{\circ}
\end{aligned}
$$

The translation represents the intuitions explained earlier in this section.

A term with a typing of the form $x_{1}: A_{1}, \ldots, x_{n}: A_{n} \triangleright M: C$ is translated to a relation $R$ of type $\mathbb{I F} A_{1}^{0} \times \ldots \times \mathbb{F} A_{n}^{\circ} \leftrightarrow C^{\circ}$. We denote such relations using the notation $\bar{t}|\psi| u$ where $\bar{t}$ is a tuple of terms of type $\mathbb{F F} A_{1}^{0} \times \ldots \times \mathbb{F} A_{n}^{\circ}, u$ a term of type $C^{\circ}$ and $\psi$ a formula. This is meant to denote a relation $R$ defined by

$$
R\left(x_{1}, \ldots, x_{n}, u\right) \leftarrow x_{1}=t_{1}, \ldots, x_{n}=t_{n}, \psi
$$

The translation will be defined by induction on type derivations. For each type rule, we give a translation rule in terms of relation triples. Using these one can construct a derivation tree of relations, each relation corresponding to a PCF term in the type derivation. Each use of the mkgraph primitive generates a recursive predicate definition. The collection of such predicate definitions forms 
Table 1. Definition of PCF

$$
\begin{aligned}
& \text { Types : } A, B, C \\
& A::=\text { int } \mid A_{1} \rightarrow A_{2} \\
& \text { Variables : } x, y, z \\
& \text { Numbers : } n::=0|1| \ldots \\
& \text { Terms : } M, N, V \\
& M::=x|n| M+N|M-N| \text { if } M N_{1} N_{2}|\lambda x . M| M N \mid \text { rec } M \\
& \text { Type rules : } \\
& \frac{\Gamma, x: A, y: B, \Gamma^{\prime} \triangleright M: C}{\Gamma, y: B, x: A, \Gamma^{\prime} \triangleright M: C} \quad \frac{\Gamma, y_{1}: A, y_{2}: A \triangleright M: C}{\Gamma, x: A \triangleright M\left[x / y_{1}, x / y_{2}\right]: C} \quad \frac{\Gamma \triangleright M: C}{\Gamma, x: A \triangleright M: C} \\
& \overline{x: A \triangleright x: A} \quad \frac{\Gamma \vdash M: A \quad \Delta, x: A \vdash N: C}{\Gamma, \Delta \vdash N[M / x]: C} \\
& \frac{\Gamma \triangleright M: \text { int } \Delta \triangleright N: \text { int }}{\Gamma, \Delta \triangleright M+N: \text { int }} \quad \frac{\Gamma \triangleright N_{1}: A \quad \Gamma \triangleright N_{2}: A}{\Gamma, x: \text { int } \triangleright \text { if } x N_{1} N_{2}: A} \\
& \triangleright n: \text { int } \\
& \frac{\Gamma, x: A \triangleright M: B}{\Gamma \triangleright \lambda x . M: A \rightarrow B} \quad \frac{\Gamma \triangleright M: A \rightarrow B}{\Gamma, x: A \triangleright M x: B} \\
& x: A \rightarrow A \triangleright \operatorname{rec} x: A
\end{aligned}
$$

a logic program and the relation triple corresponding to the overall term becomes the query.

The translations for the first three rules (called the structural rules of Exchange, Contraction and Weakening) are straightforward:

$$
\begin{array}{cc}
\frac{\Gamma, x: A, y: B, \Gamma^{\prime} \triangleright M: C}{\Gamma, y: B, x: A, \Gamma^{\prime} \triangleright M: C} & \frac{\bar{t}, u_{1}, u_{2}, \bar{t}^{\prime}|\psi| v}{\bar{t}, u_{2}, u_{1}, \bar{t}^{\prime}|\psi| v} \\
\frac{\bar{T}, y_{1}: A, y_{2}: A \triangleright M: C}{\Gamma, x: A \triangleright M\left[x / y_{1}, x / y_{2}\right]: C} & \frac{\bar{t}, u_{1}, u_{2}|\psi| v}{\bar{t}, u_{1} \cup u_{2}|\psi| v} \\
\frac{\Gamma \triangleright M: C}{\Gamma, x: A \triangleright M: C} & \frac{\bar{t}|\psi| v}{\bar{t},\{\}|\psi| v}
\end{array}
$$

The next two rules (called Identity and Cut) are translated as follows:

$$
\begin{array}{cc}
\overline{x: A \triangleright x: A} & \overline{\{z\} \mid \text { true } \mid z} \\
\frac{\Gamma \vdash M: A \quad \Delta, x: A \vdash N: C}{\Gamma, \Delta \vdash N[M / x]: C} & \frac{\bar{t}_{1}\left|\psi_{1}\right| u \quad \bar{t}_{2}, u^{\prime}\left|\psi_{2}\right| v}{\bar{x}_{1}, \bar{t}_{2}\left|\mathbf{m k g r a p h}\left[\bar{t}_{1}\left|\psi_{1}\right| u\right]\left(\bar{x}_{1}, u^{\prime}\right), \psi_{2}\right| v}
\end{array}
$$

The complexity of the Cut rule is due to the fact that the term $u$ in the first premise is of type $A^{\circ}$ whereas $u^{\prime}$ in the second premise is of type IF $A^{\circ}$. Therefore, we must generate a graph of $u$ 's and match it to $u^{\prime}$. 
The primitives are translated as follows:

$$
\begin{array}{cc}
\begin{array}{c}
\Delta n: \text { int } \\
\Gamma \triangleright M: \text { int } \Delta \triangleright N: \text { int }
\end{array} & \frac{\bar{t}_{1}\left|\psi_{1}\right| u_{1} \bar{t}_{2}\left|\psi_{2}\right| u_{2}}{\mid \Gamma, \Delta \triangleright M+N: \text { int }} \\
\frac{\Gamma \triangleright N_{1}: A \quad \Gamma \triangleright N_{2}: A}{\Gamma, x: \text { int } \triangleright \text { if } x N_{1} N_{2}: A} & \frac{\bar{t}_{1}, \bar{t}_{2}\left|\psi_{1}, \psi_{2}, \operatorname{add}\left(u_{1}, u_{2}, z\right)\right| z}{x,\{x\}\left|\begin{array}{l}
\left(x=0, \bar{x}=\bar{t}_{1}, \psi_{1}, y=v_{1}\right) \vee \\
\left(x \neq 0, \bar{x}=\bar{t}_{2}, \psi_{2}, y=v_{2}\right)
\end{array}\right| y}
\end{array}
$$

Notice that the translation of the conditional gives rise to a disjunction. In a concurrent logic programming language, one must treat $x=0$ and $x \neq 0$ as guards of the two branches.

Finally, the higher-order terms are translated by:

$$
\begin{array}{cc}
\frac{\Gamma, x: A \triangleright M: B}{\Gamma \triangleright \lambda x . M: A \rightarrow B} & \frac{\bar{t}, u|\psi| v}{\bar{t}|\psi|(u, v)} \\
\frac{\Gamma \triangleright M: A \rightarrow B}{\Gamma, x: A \triangleright M x: B} & \frac{\bar{t}|\psi| u}{\bar{t}, X|\psi, u=(X, z)| z} \\
\hline x: A \rightarrow A \triangleright \operatorname{rec} x: A & \overline{F|\operatorname{recurse}(F, z)| z}
\end{array}
$$

\begin{tabular}{|c|c|c|c|}
\hline$x:$ int $\triangleright x:$ int & $\triangleright 1:$ int & $\{x\} \mid$ true $\mid x$ & | true | 1 \\
\hline \multicolumn{2}{|c|}{$x:$ int $\triangleright x+1:$ int } & \multicolumn{2}{|c|}{$x$}$|\operatorname{add}(x, 1, y)| y$ \\
\hline$\triangleright \lambda x \cdot x+$ & & $\operatorname{add}(x, 1, y)$ & $\overline{(\{x\}, y)}$ \\
\hline
\end{tabular}

Notice that abstraction is pair formation and application is pair decomposition. The recurse predicate used in the last rule is defined as follows:

$$
\begin{aligned}
& \text { recurse }: \mathbb{F}(\mathbb{F} A \times A) \leftrightarrow A \\
& \operatorname{recurse}(\mathbf{F}, \mathbf{x}) \leftarrow \mathrm{F}=\{(\mathrm{X}, \mathrm{x})\} \cup \mathrm{F}^{\prime}, \operatorname{mkgraph}[\mathrm{F}|\operatorname{recurse}(\mathrm{F}, \mathrm{x})| \mathrm{x}]\left(\mathrm{F}^{\prime}, \mathrm{X}\right)
\end{aligned}
$$

As an example of the translation, consider the succ function:

The translation triple of this term denotes a relation succ : IF int $\leftrightarrow$ int defined by:

$$
\operatorname{succ}((\{x\}, y)) \leftarrow \operatorname{add}(x, 1, y)
$$

This differs from the translation given at the beginning of Sec. 3 in that there we did not model the input of succ as of type IF int. The translation given here treats all types uniformly. But, int being a pure data type with no higher-order values, the use of IF can be dropped. This and other optimizations are discussed in Sec. 3.3 below.

We show the correctness of the translation as follows: 
Lemma 2 (type soundness). The translation of a PCF term $x_{1}: A_{1}, \ldots, x_{n}$ : $A_{n} \triangleright M: C$ is a relation of type $\mathbb{F} A_{1}^{\circ} \times \ldots \times \mathbb{F} A_{n}^{\circ} \leftrightarrow C^{\circ}$.

Theorem 3. The following equivalences of lambda terms are preserved by the translation:

$$
\begin{aligned}
(\lambda x . M) N & \equiv M[N / x] \\
\lambda x . M x & \equiv M \\
\operatorname{rec} M & \equiv M(\operatorname{rec} M)
\end{aligned}
$$

Proof. For $(\lambda x . M) N$ we have a translation of the following form:

$$
\begin{aligned}
& \frac{\Delta, x: A \triangleright M: B}{\Delta \triangleright \lambda x \cdot M: A \rightarrow B} \\
& \frac{\Gamma \triangleright N: A \quad \overline{\Delta, y: A \triangleright(\lambda x . M) y: B}}{\Gamma, \Delta \triangleright(\lambda x . M) N: B} \\
& \begin{array}{c}
\frac{\bar{t}, u|\psi| v}{\bar{t}|\psi|(u, v)} \\
\overline{\bar{t}^{\prime}\left|\psi^{\prime}\right| u^{\prime}} \frac{\bar{t}, X|\psi,(u, v)=(X, z)| z}{\bar{x}^{\prime}, \bar{t}\left|\mathbf{m k g r a p h}\left[\bar{t}^{\prime}\left|\psi^{\prime}\right| u^{\prime}\right]\left(\bar{x}^{\prime}, X\right), \psi,(u, v)=(X, z)\right| z}
\end{array}
\end{aligned}
$$

By unifying $(u, v)$ with $(X, z)$ and substituting for $X$ and $z$, we obtain the translation of $M[N / x]$ :

$$
\frac{\Gamma \vdash N: A \quad \Delta, x: A \vdash M: C}{\Gamma, \Delta \vdash M[N / x]: C} \frac{\bar{t}^{\prime}\left|\psi^{\prime}\right| u^{\prime} \quad \bar{t}, u|\psi| v}{\bar{x}^{\prime}, \bar{t}\left|\operatorname{mkgraph}\left[\bar{t}^{\prime}\left|\psi^{\prime}\right| u^{\prime}\right]\left(\bar{x}^{\prime}, u\right), \psi\right| v}
$$

The preservation of the eta equivalence follows similarly from the properties of unification and the recursion equivalence follows from the definition of the recurse predicate.

\subsection{Data structures}

The translation can be readily extended to deal with data structures. Product and sum types of a functional language are translated as follows:

$$
\begin{aligned}
u n i t^{\circ} & =\emptyset \\
(A \times B)^{\circ} & =\mathrm{fst}\left(A^{\circ}\right)+\operatorname{snd}\left(B^{\circ}\right) \\
(c(A)+d(B))^{\circ} & =c\left(\mathrm{FF} A^{\circ}\right)+d\left(\mathrm{~F} B^{\circ}\right)
\end{aligned}
$$

In the case of product types, fst and snd are assumed to be constructor symbols not used elsewhere. Notice that the translation of product types exhibits the same kind of reduction in the cardinality as encountered with function types previously. Think of a pair-typed value as a process that responds to fst and snd messages. 
The translations of terms can be easily derived using the translation of types as a heuristic. For binary products:

$$
\frac{\Gamma \triangleright M: A \quad \Gamma \triangleright N: B}{\Gamma \triangleright(M, N): A \times B} \frac{\bar{t}_{1}\left|\psi_{1}\right| u_{1} \bar{t}_{2}\left|\psi_{2}\right| u_{2}}{\bar{x}\left|\begin{array}{l}
\left(y=\mathrm{fst}\left(u_{1}\right), \bar{x}=\bar{t}_{1}, \psi_{1}\right) \vee \\
\left(y=\operatorname{snd}\left(u_{2}\right), \bar{x}=\bar{t}_{2}, \psi_{2}\right)
\end{array}\right| y}
$$

The relation for the pair has two clauses. One of them will get exercised when a component is selected. Similarly, the translation of terms of sum types is:

$$
\begin{aligned}
& \frac{\Gamma \triangleright M: A}{\Gamma \triangleright c(M): c(A)+d(B)} \quad \frac{\bar{t}|\psi| u}{\bar{t}|\psi| c(u)} \\
& \Gamma, x_{1}: A \triangleright M: C \quad \Gamma, x_{2}: B \triangleright N: C \\
& \overline{\Gamma, x: c(A)+d(B) \triangleright \text { case } x \text { of } c\left(x_{1}\right) \Rightarrow M \mid d\left(x_{2}\right) \Rightarrow N: C} \\
& \frac{\bar{t}_{1}, t_{1}\left|\psi_{1}\right| u_{1} \quad \bar{t}_{2}, t_{2}\left|\psi_{2}\right| u_{2}}{\bar{x},\{x\}\left|\begin{array}{l}
\left(x=c\left(t_{1}\right), \bar{x}=\bar{t}_{1}, z=u_{1}\right) \vee \\
\left(x=d\left(t_{2}\right), \bar{x}=\bar{t}_{2}, z=u_{2}\right)
\end{array}\right| z}
\end{aligned}
$$

Many data structures occurring in practice take the form of sum-of-products. For example, lists are given by a type definition of the form:

type list $=$ nil $($ unit $)+\operatorname{cons}($ int $\times$ list $)$

In translating such a type to the logic programming context, we can make use of the isomorphisms:

$$
\mathbb{F}(A+B) \cong \mathbb{F} A \times \mathbb{F} B \quad \mathbb{F} \emptyset \cong \text { unit }
$$

So, the translation of the list type can be given as:

$$
\operatorname{list}^{\circ}=\operatorname{nil}(\text { unit })+\operatorname{cons}\left(\mathbb{F} \text { int } \times \mathbb{F} \text { list }^{\circ}\right)
$$

\subsection{Optimizations}

The type IFint denotes finite sets of integers. Since functional programs give determinate results, such a set contains at most one integer. The empty set, \{\} , appears when a program ignores its integer input. A singleton set appears when the program uses the input. The use of finite sets for data values thus provides "lazy" data. Similarly, the above type list $^{\circ}$ makes available lazy lists within a concurrent logic program.

If we are not interested in lazy data structures, we can eliminate all uses of IF in data structures. To model this, we use the following translation:

$$
\begin{aligned}
\text { int }^{*} & =\text { int } \\
\text { unit } & =u n i t \\
(A \times B)^{*} & =A^{*} \times B^{*} \\
(c(A)+d(B))^{*} & =c\left(A^{*}\right)+d\left(B^{*}\right)
\end{aligned}
$$

For example, 


$$
\text { list* }=\text { nil(unit })+ \text { cons }\left(\text { int } \times \text { list }^{*}\right)
$$

The function type $A \rightarrow B$ is then translated to $A^{*} \times B^{\circ}$ instead of $\mathbb{F} A^{\circ} \times B^{\circ}$. We will see applications of this translation in the next section.

Another opportunity for optimization occurs when the argument of a function is used linearly, i.e., if the function uses its argument precisely once. In this case, we can simplify the translation of functions to

$$
(A \rightarrow B)^{\circ}=A^{\circ} \times B^{\circ}
$$

This optimization is already illustrated in the examples at the beginning of Sec. 3 .

\subsection{Linear logic}

The reader familiar with linear logic would notice that our translation closely parallels Girard's embedding of intuitionistic logic in linear logic [Gir87]. Indeed, our debt to linear logic is considerable. Logic programming (more precisely, the category of sets and relations) forms a model of linear logic: \& and $\oplus$ are interpreted as disjoint union, $\otimes$ and $\rightarrow$ are interpreted as product, and ! is interpreted as finite sets. The translation given above is essentially a restatement of Girard's translation in this new light.

\section{Example applications}

The results of Sec. 3 establish a close correspondence between the higher-order programming techniques of functional programming and those of logic programming. Two features of logic programming play a role in this correspondence:

- logic variables, and

- concurrency.

The higher-order programming techniques are available only to a limited extent in sequential logic programming due to the crucial role of concurrency in this correspondence. However, they are still available and logic programmers frequently make use of them. In this section, we draw a correspondence between some of the common programming techniques used in functional and logic programming paradigms in the light of our translation. We hope that this will lead to a reexamination of the logic programming techniques in the light of "higher-order programming" and pave the way for further exchange of ideas and techniques between the two paradigms.

Difference lists form a familiar logic programming technique which allow for constant time concatenation [CT77]. A very similar technique was developed in functional programming using function values [Hug86]. Our correspondence identifies the two techniques, i.e., if we translate the functional programming representation of difference lists using the translation of Sec. 3, we obtain the logic programming representation. 
The functional programming technique is shown in Table 2. A difference list is represented as a function from lists to lists. The representation of an ordinary list $\left[x_{1}, \ldots, x_{n}\right]$ is the function $\lambda l . x_{1}, x_{2} \ldots \ldots x_{n} . l$. (See the function $r e p$.) Concatenation is then just function composition, which works in constant time.

Table 2. Difference lists in functional programming

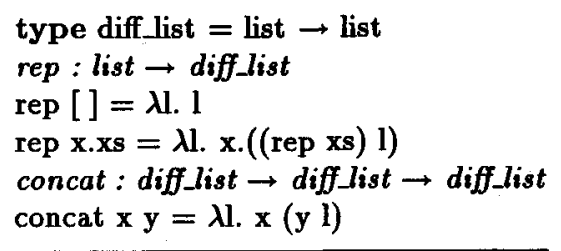

The logic programming technique is shown in Table 3. Here, a difference list is a pair of lists, but one of the lists is typically a logic variable which is shared in the other list. The representation of a list $\left[x_{1}, \ldots, x_{n}\right]$ is a pair $\left(x_{1} . x_{2} \ldots \ldots x_{n} . l, l\right)$ where $l$ is a variable.

Table 3. Difference lists in logic programming

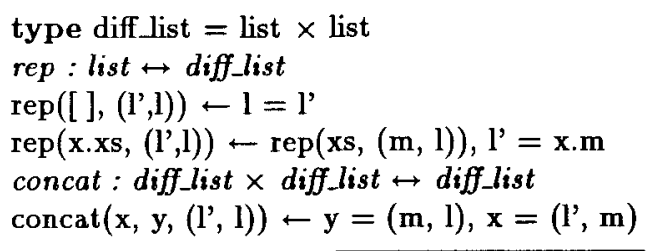

To see the correspondence between the two versions, notice that the functional difference list uses its list argument linearly. Thus, as noted in Sec. 3.3, list $\rightarrow$ list can be translated to list $\times$ list in the logic programming context. (The second component corresponds to the input list and the first component to the output list.) A close examination of the two programs is quite striking. They correspond operator by operator according to the translation (3).

A difference structure, in general, involves some data or computational structure with a place holder inside the structure captured by a logic variable. That is essentially what a function is. It is a computation with a place holder for the argument. Thus, the example we found here is an instance of a general concept.

Difference lists of Table 3 can only be used linearly. Our technique also allows us to build difference lists that can be used multiple times. For example, 


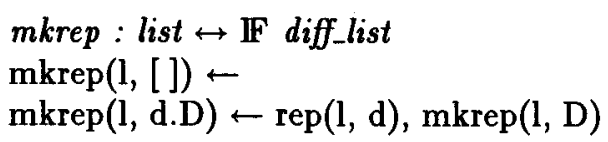

allows a copy of a difference list to be produced on demand. To check if a difference list is empty, we can then use a goal of the form:

$$
D=d \cdot D^{\prime}, d=(l,[]), l=[]
$$

This kind of a goal has the problem that it makes an entire copy of a difference list to check if it is empty. We can optimize it by using lazy lists instead of standard lists. (Cf. Sec. 3.3).

Another important application for function values is in abstract syntax. Consider a language that has variable-binding operators, e.g., lambda calculus or predicate calculus. In writing a processor for such a language (evaluator, theorem prover etc.), there arise delicate issues of variable renaming, substitution etc. Pfenning and Elliott [PE88] proposed that such issues can be treated in modular fashion by using higher-order abstract syntax, i.e., syntactic representations that involve function values. To see how this works, consider the first-order and higher-order representations for lambda terms:

$$
\begin{aligned}
& \text { term }=\operatorname{var}(\text { symbol })+\operatorname{lam}(\text { symbol } \times \text { term })+\text { ap }(\text { term } \times \text { term }) \\
& \text { hoterm }=\operatorname{lam}(\text { hoterm } \rightarrow \text { hoterm })+\operatorname{ap}(\text { hoterm } \times \text { hoterm })
\end{aligned}
$$

The higher-order representation (hoterm) is symbol-free. The variable binding operator, lam, takes a function mapping terms (the arguments) to terms (the result of embedding the argument in the body of the abstraction). For example, the Church numeral $2=\lambda s \cdot \lambda x \cdot s(s x)$, has the following representations:

first-order: $\operatorname{lam}(" s ", \operatorname{lam}(" x ", \operatorname{ap}(\operatorname{var}(" s "), \operatorname{ap}(\operatorname{var}(" s "), \operatorname{var}(" x ")))))$ higher-order: $\operatorname{lam}(\lambda t \operatorname{lam}(\lambda u . \operatorname{ap}(t, \operatorname{ap}(t, u))))$

Table 4 shows a parser that converts a first-order representation to the higherorder one and a normalizer that operates on higher-order representations. Notice that substitution is handled cleanly in the definition of apply $\operatorname{lam}(f) n$. The result of substituting $n$ for the formal parameter is simply $f n$. In effect, all substitution is performed at parse-time with the result that processing of the language is considerably simplified.

Precisely the same effect can be achieved in logic programming. One can replace all the occurrences of symbols by logic variables so that substitution is achieved by simply binding the logic variables. This technique has been used, for instance, in the polymorphic type inference algorithm of Typed Prolog [LR91, Lak91]. We would like to argue that this is not merely achieving the same effect as higher-order abstract syntax, but it is in fact the same technique. It is an instance of our translation. However, the standard technique used in logic programming is not as general as the higher-order technique. In particular, it does not work if the terms substituted for variables have bound variables. In that case, one has to rename bound variables with fresh logical variables and the cleanliness of 
Table 4. Higher-order abstract syntax in functional programming

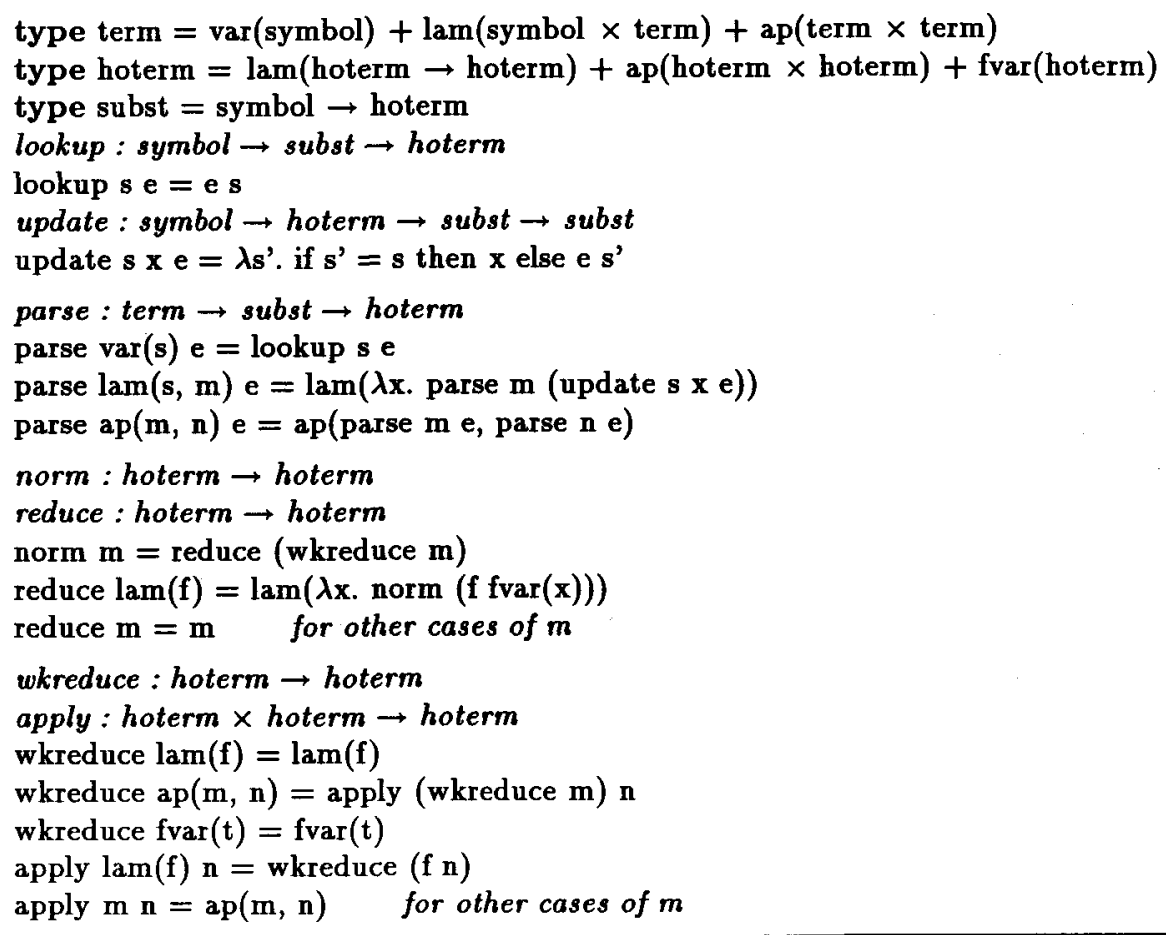

the technique is compromised. On the other hand, our translation handles the general case as well. The naive logic programming technique is the special case where inputs of functions are assumed to be data objects with no embedded functions.

In Table 5, we show the naive logic programming technique for a normalizer of lambda terms. Notice that lambda abstractions are represented by terms of type lam(hoterm $\times$ hoterm). When an abstraction $\lambda s . m_{s}$ is converted to this representation, a fresh logical variable $x$ is created and all occurrences of the symbol $s$ are replaced with $x$. See the clause for $\operatorname{parse}(\operatorname{lam}(s, m), e, t)$. The resulting representation is $\operatorname{la} m\left(x, m_{x}\right)$. To reduce an application of such an abstraction term, in the clause $\operatorname{apply}(\operatorname{lam}(f), n, t)$, we bind $x$ to the argument term $n$ and reduce $m_{n}$.

The point is that the logic programming representation lam (hoterm $\times$ hoterm) is a naive encoding of the functional representation. It works in the following cases:

- every $m_{x}$ contains precisely one occurrence of $x$, i.e., lambda terms are linear, or 
Table 5. A naive logic program for higher-order abstract syntax

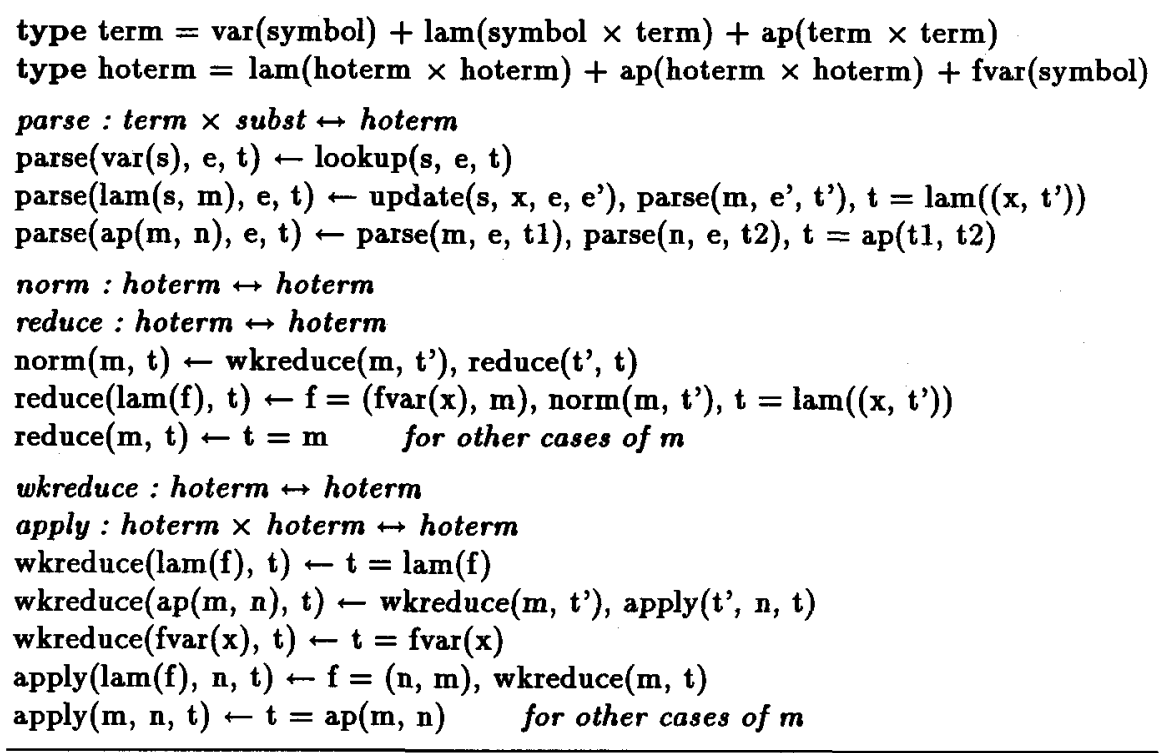

- the argument term has no bound variables, i.e., argument terms are pure data.

These two cases correspond to the translation schemes (3) and (2). To handle the general case, we must use the general translation scheme (1). The result is shown in Table 6. We have used the optimization of treating term and symbol as pure data. Moreover, the arguments of norm, reduce and wkreduce and the first argument of apply are treated as linear inputs. These optimizations give considerable simplifications, but the reader might still find the resulting program hard to comprehend. One would want to devise usable syntactic mechanisms to cut down this complexity. (The focus of this paper being the expressive power of pure logic programming, we have refrained from doing so.)

In this program, mkparse is used to generate multiple hoterm's for any given first-order term. Each hoterm receives a fresh set of logic variables to represent its bound variables. (See parselam.) No extra mechanism for variable renaming is necessary.

Many other programming techniques of higher-order functional programming can be similarly adapted to the logic programming context. See [BW88, Pau91, Wad92]. The reader is invited to try some of these using the translation scheme of Section 3. 
Table 6. A general logic program for higher-order abstract syntax

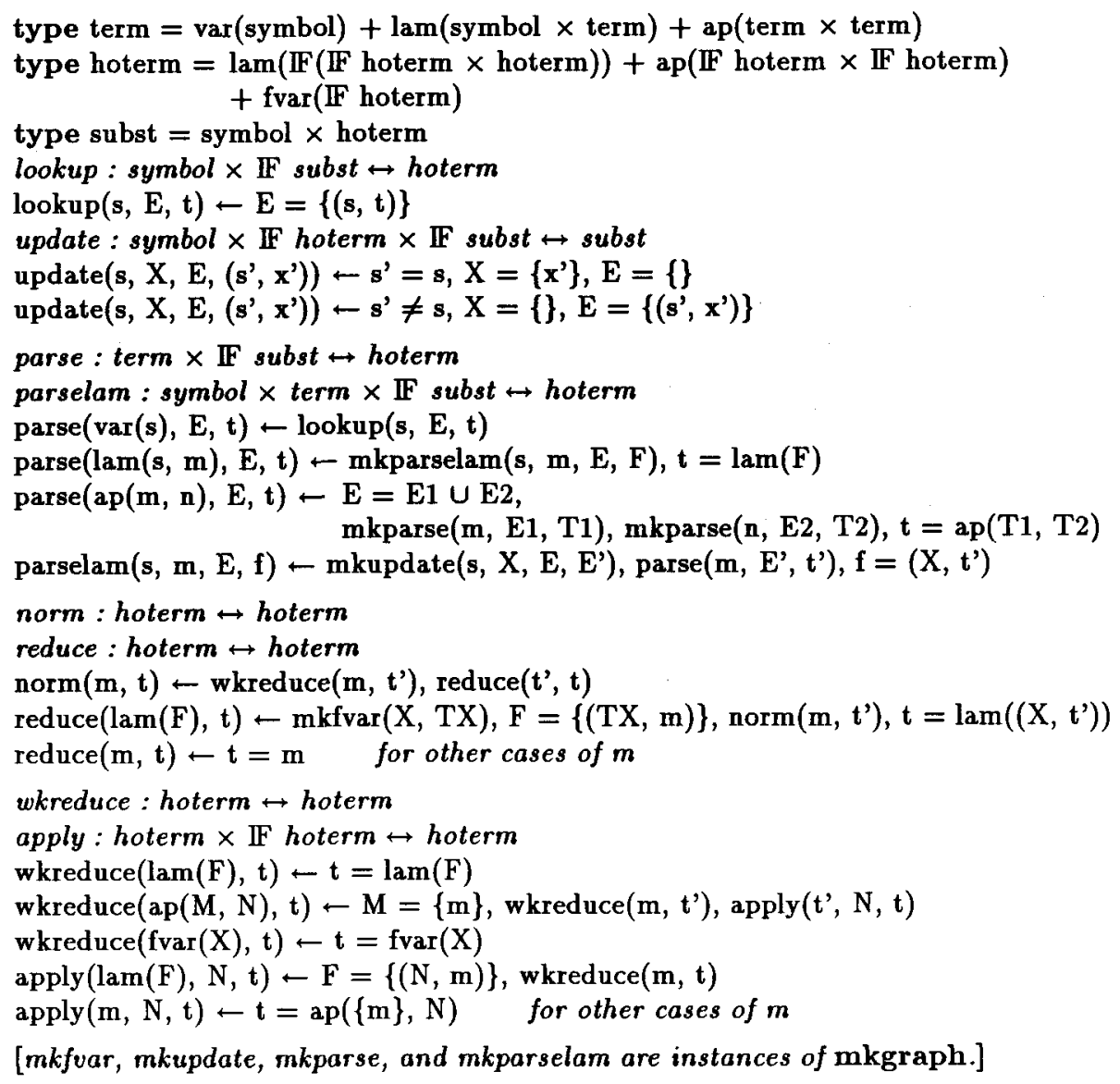

\section{Conclusion}

We have demonstrated the expressive power of logic programming by modelling higher-order programming features in pure logic programs. Further, these programming techniques, in a limited context, correspond to well-known techniques of logic programs.

However, the significant abstraction facilities made possible by higher-order techniques are not available in sequential logic programming. Thus, we believe that sequential logic programming is a poor approximation to logic programming and that efforts must be made to incorporate concurrency and fairness. Concurrent logic programming languages as well as coroutining facilities [Nai86] are an important step in this direction. These systems need to be extended to deal with backtracking nondeterminism. Formal semantics and type systems must be 
developed to place them on a firm foundation.

The correspondences drawn in this work are only a first step in exploiting the richness of the logic programming paradigm. These techniques must be further explored and applied to practical contexts.

\section{References}

[Abr91] S. Abramsky. Computational interpretation of linear logic. Tutorial Notes, International Logic Programming Symposium, San Diego, 1991, 1991.

[Abr93] S. Abramsky. Computational interpretations of linear logic. Theoretical Comp. Science, 111(1-2):3-57, 1993.

[Bar91] M. Barr. *-Autonomous categories and linear logic. Math. Structures in Comp. Science, 1:159-178, 1991.

[BW88] R. Bird and P. Wadler. Introduction to Functional Programming. PrenticeHall International, London, 1988.

[CKW89] W. Chen, M. Kifer, and D. S. Warren. HiLog: A first-order semantics for higher-order logic programming constructs. In L. Lusk, $\mathrm{E}$ and $\mathrm{R}$. A. Overbeek, editors, Logic Programming: Proc. of the North American Conf. 1989, pages 1090-1144. MIT Press, 1989.

[CT77] K. L. Clark and S. A. Tarnlund. A first-order theory of data and programs. In Information Processing, pages 939-944. North-Holland, 1977.

[DOPR91] A. Dovier, E. G. Omodeo, E. Pontelli, and G. F. Rossi. \{log\}: A logic programming langugae with finite sets. In K. Furukawa, editor, Logic Programming: Proceedings of the Eigth International Conference. MIT Press, 1991.

[Gir87] J.-Y. Girard. Linear logic. Theoretical Comp. Science, 50:1-102, 1987.

[Gre87] S. Gregory. Parallel Logic Programming in PARLOG: The Language and its Implementation. Addison-Wesley, Reading, Mass., 1987.

[Gun92] C. A. Gunter. Semantics of Programming Languages: Structures and Techniques. MIT Press, 1992.

[Hug86] R. J. M. Hughes. A novel representation of lists and its application to the function "reverse". Information Processing Letters, 22:141-144, Mar 1986.

[Laf87] Y. Lafont. Linear logic programming. In P. Dybjer, editor, Porc. Workshop on Programming Logic, pages 209-220. Univ. of Goteborg and Chalmers Univ. Technology, Goteborg, Sweden, Oct 1987.

[Lak91] T. K. Lakshman. Typed prolog: Type checking/type reconstruction system (version 1.0). Software available by anonymous FTP from cs.uiuc.edu, 1991.

[LM84] J.-L. Lassez and M. J. Maher. Closures and fairness in the semantics of programming logic. Theoretical Computer Science, pages 167-184, May 1984.

[LR91] T.K. Lakshman and U.S. Reddy. Typed Prolog: A Semantic Reconstruction of the Mycroft-O'Keefe Type System. In V. Saraswat and K. Ueda, editors, Logic Programming: Proceedings of the 1991 International Symposium, pages 202 - 217. MIT Press, Cambridge, Mass., 1991.

[Mil90] R. Milner. Functions as processes. In Proceedings of ICALP 90, volume 443 of Lect. Notes in Comp. Science, pages 167-180. Springer-Verlag, 1990.

[MN86] D. A. Miller and G. Nadathur. Higher-order logic programming. In Intern. Conf. on Logic Programming, 1986. 
[Nai86] Lee Naish. Negation and control in Prolog, volume 238 of Lect. Notes in Comp. Science. Springer-Verlag, New York, 1986.

[Pau91] L. C. Paulson. ML for the Working Programmer. Cambridge Univ. Press, Cambridge, 1991.

[PE88] F. Pfenning and C. Elliott. Higher-order abstract syntax. In $A C M$ SIGPLAN '88 Conf. Program. Lang. Design and Impl., pages 22-24. ACM, 1988.

[Plo77] G. D. Plotkin. LCF considered as a programming language. Theoretical Comp. Science, 5:223-255, 1977.

[Red93a] U.S. Reddy. Higher-order functions and state-manipulation in logic programming. In R. Dyckhoff, editor, Fourth Workshop on Extensions of Logic Programming, pages 115-126, St. Andrews, Scotland, Mar 1993. St. Andrews University.

[Red93b] U.S. Reddy. A typed foundation for directional logic programming. In E. Lamma and P. Mello, editors, Extensions of Logic Programming, volume 660 of Lect. Notes in Artificial Intelligence, pages 282-318. Springer-Verlag, 1993.

[Sar92] Vijay Saraswat. The category of constraint systems is Cartesian-closed. In Proceedings, Seventh Annual IEEE Symposium on Logic in Computer Science, pages 341-345, Santa Cruz, California, 22-25 June 1992. IEEE Computer Society Press.

[Sco69] D. S. Scott. A type theoretical alternative to CUCH, ISWIM and OWHY. Unpublished manuscript, Oxford University, 1969.

[Sha83] E. Y. Shapiro. A subset of Concurrent Prolog and its interpreter. Technical Report TR-003, ICOT- Institute of New Generation Computer Technology, January 1983. (Reprinted in [Sha87].).

[Sha87] E. Shapiro. Concurrent Prolog: Collected Papers. MIT Press, 1987. (Two volumes).

[ST86] E. Shapiro and A. Takeuchi. Object-oriented programming in Concurrent Prolog. New Generation Computing, 4(2):25-49, 1986. (reprinted in [Sha87].).

[Ued86] K. Ueda. Guarded Horn clauses. In E. Wada, editor, Logic Programming, pages 168-179. Springer-Verlag, 1986. (reprinted in [Sha87].).

[Wad92] P. Wadler. The essence of functional programming. In ACM Symp. on Princ. of Program. Lang., 1992.

[War82] D. H. D. Warren. Higher-order extensions to Prolog: Are they needed? In D. Michie, editor, Machine Intelligence, 10, pages 441-454. Edinburgh University Press, 1982. 